\title{
SONOS Nonvolatile Memory Cell Programming Characteristics
}

\author{
TODD C. MACLEOD ${ }^{\mathrm{a}}$, THOMAS A. PHILLIPS ${ }^{\mathrm{a}}$, and FAT D. HO \\ ${ }^{\mathrm{a}}$ National Aeronautics and Space Administration, Marshall Space Flight Center, \\ Huntsville, Alabama, 35812, U.S.A. \\ ${ }^{\mathrm{b}}$ The University of Alabama in Huntsville, Department of Electrical and Computer Engineering, \\ Huntsville, Alabama 35899, U.S.A.
}

\begin{abstract}
Silicon-oxide-nitride-oxide-silicon (SONOS) nonvolatile memory is gaining favor over conventional EEPROM FLASH memory technology. This paper characterizes the SONOS write operation using a nonquasi-static MOSFET model. This includes floating gate charge and voltage characteristics as well as tunneling current, voltage threshold and drain current characterization. The characterization of the SONOS memory cell predicted by the model closely agrees with experimental data obtained from actual SONOS memory cells. The tunnel current, drain current, threshold voltage and read drain current all closely agreed with empirical data.
\end{abstract}

Keywords: SONOS, MOSFET Model, FLASH 

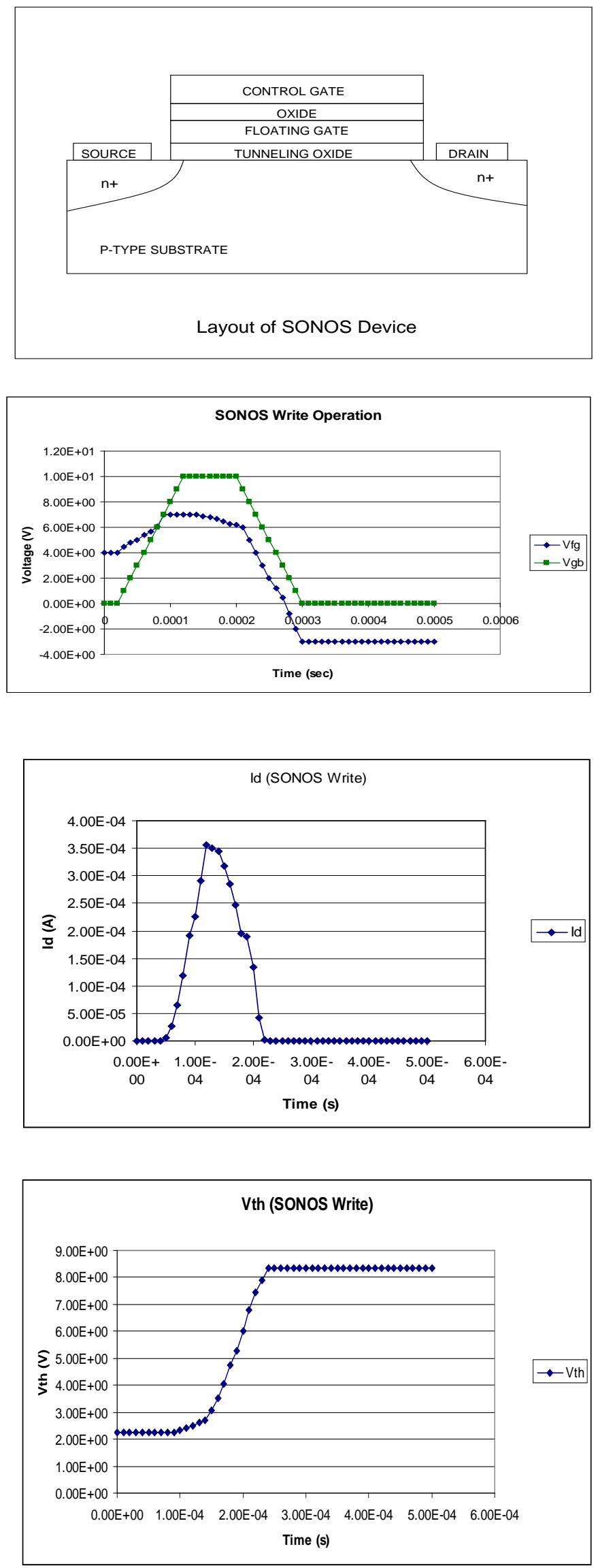